\title{
Ciudad Vieja; un modelo para armar.
}

\author{
Old City; a puzzle to solve.
}

\author{
Arq. Paulo Adhemar Pereyra Bonifácio \\ Facultad de Arquitectura, Diseño y Urbanismo \\ Universidad de la República, Uruguay \\ paulopereyra@fadu.edu.uy
}

\begin{abstract}
This research arises from the realization of a model from an historic district by digital fabrication. Seeks to think about and document the integration of the potentialities of Applied Informatics and digital manufacturing processes, of urban revitalization through social responsibility projects. This issue contributes to sustainable and intelligent urban development, supporting initiatives ad hoc through the incorporation of collaborative processes, distributed intelligence and team research.
\end{abstract}

Keywords: Digital fabrication; Applied informatics, intelligent urban development, distributed intelligence.

\section{Introducción}

El presente trabajo de investigación, que surge a partir de la oportunidad que genera el pedido de realización de una maqueta de un barrio histórico- Ciudad Vieja; la ciudad fundacional en la que se asentó la colonización española en Uruguay- mediante tecnologías de Fabricación Digital, busca reflexionar, y documentar, la integración de las potencialidades de la Informática Aplicada en proyectos de responsabilidad social que contribuyan al desarrollo sostenible e inteligente de las ciudades apoyando iniciativas ad hoc a través de la integración en procesos colaborativos , de inteligencia distribuida, de investigación colectiva. Dicho trabajo de investigación se implementó mediante un convenio entre la Intendencia de Montevideo y la Facultad de Arquitectura, a través del Deplnfo (Departamento de Informática Aplicada al Diseño), en particular a sus dos laboratorios; VidiaLab-laboratorio de visualización digital avanzada- y LabFab- laboratorio de fabricación digital).-

Figura 01

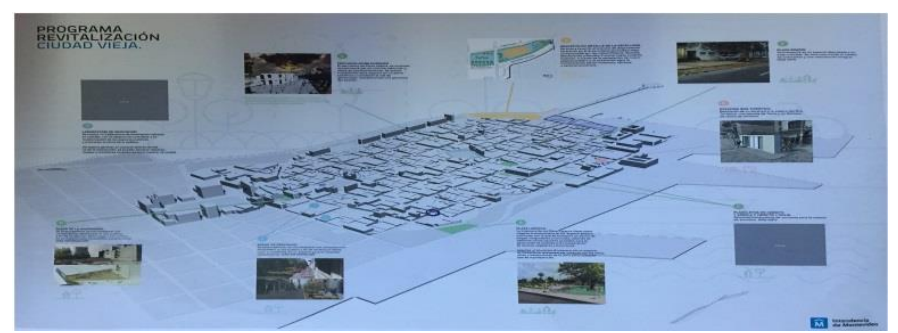

\section{Modelo para armar;}

La motivación de la Intendencia de Montevideo, más allá de la materialización de una maqueta, es armar un modelo sobre el que visualizar las intervenciones urbano-arquitectónica que, actualmente, está llevando a cabo para revitalizar el centro histórico desde una impronta sustentable e inteligente. Dicho modelo será uno de los instrumentos que formarán parte en la implementación de un Laboratorio de Innovación ciudadana que se constituirá en el Cabildo de Montevideo. Para ello ha implementado un Programa de Revitalización Urbana, que tiene como marco de referencia al Plan de Ordenación, Participación y Mejora de la Ciudad Vieja, aprobado en el año 2003. Se inscribe dentro de una serie de iniciativas que impulsan la extensión de las superficies peatonales, la revitalización de los espacios públicos más significativos y la revalorización del patrimonio edilicio. El Programa está compuesto de siete subprogramas físicos (cuya finalidad es la calificación de los espacios públicos de la zona mediante la implementación de una serie de intervenciones urbanas) y nueve subprogramas de gestión (que apuntan a dotar a dichas intervenciones urbanas de una integralidad de contenidos temáticos considerados indispensables). Cada subprograma ofrece una serie de productos (intervenciones urbanasarquitectónicas) a los que llega a través del desarrollo de proyectos específicos; que apuntan a la recuperación y recambio del pavimento de las veredas en mal estado, la incorporación de rampas en las esquinas, el cambio de equipamiento urbano (papeleras, bolardos, paradas de ómnibus), nueva cartelería del nomenclator, señales de tránsito, cambio y ampliación del sistema de iluminación, ensanchamiento de varias veredas, la generación de zonas de coexistencia de tráfico peatonal y vehicular, la incorporación de estaciones de descanso, la calificación de plazas y plazoletas y la incorporación de un Laboratorio de Innovación Ciudadana dentro de las instalaciones del Cabildo ( $\left.{ }^{\star} 2\right)$ de Montevideo en el que la maqueta, concebida como un modelo, será uno de los objetos a ser expuestos y tendrá una triple finalidad; por un lado será soporte de visualización de las intervenciones realizadas en el marco del Programa de Revitalización Urbana, materialización de lo ya construído y 
permitirá dar a conocer las posibilidades de las herramientas de fabricación digital. En tal sentido la maqueta actúa como un puente entre pasado, presente y futuro ya que permite visualizar el devenir edificado de las construcciones históricas, las presentes intervenciones y el mapeo de iniciativas de innovación ciudadanas generadas por el Laboratorio de Innovación Ciudadana y con aptitud para ser prototipadas mediante tecnologías de Informática Aplicada, cerrando de ese modo un círculo virtuoso.

\section{Proceso Colaborativo;}

La realización del modelo implica una "institucionalización" en el sentido de la necesidad de generar un convenio de partes, entre la institución educativa y la gubernamental, en el que se objetivice el proceso colaborativo; por un lado el "objeto" definido en sus partes, dimensiones, costos, alcances y plazos de ejecución y, por otra parte, el objeto definido como proceso en el cual se integran los distintos actores; estudiantes contratados por régimen de pasantías que, formando parte junto a los docentes e investigadores de los laboratorios universitarios en la conformación, digitalización y de materialización de la maqueta se suman a las dependencias vinculadas a la concreción del Programa entre las que se encuentran : Movilidad Urbana, Departamento de Desarrollo Sostenible e Inteligente, División Espacios Públicos y Edificaciones del Departamento de Acondicionamiento Urbano, Comisión Especial Permanente de la Ciudad Vieja, Secretaría de la Gestión de la Discapacidad del Departamento de Desarrollo Social, Municipio B (3er nivel de gobierno departamental), Departamento de Desarrollo económico e Integración regional, Gestión de Calidad y Unidad de control y Coordinación de Redes de Infraestructura Urbana.

\section{Inteligencia distribuida;}

El nuevo paradigma urbano gira en torno al concepto de "ciudad inteligente", un concepto emergente y en pleno proceso de desarrollo pero que, a grandes rasgos, podemos caracterizar como de "un ecosistema en el que coexisten múltiples procesos íntimamente ligados y que resulta difícil abordar de forma individualizada", según MIT, "Smart cities: vision" ( http://cities.media.mit.edu). Las intervenciones en las que se apoya el Programa de Revitalización Urbana, concebidas desde un enfoque transversal apuntan a una gestión basada en inteligencia distribuída en las que los distintos actores aportan desde la especificidad disciplinaria pero que tiene un objetivo general delineado y concreto: el de la sostenibilidad urbana y el empoderamiento social de la ciudadanía. Relacionado con el empoderamiento ciudadano el Laboratorio de Innovación Ciudadana; MVDLab plantea constituirse como un espacio físico y digital, un punto de encuentro interdisciplinario para el abordaje innovador de los desafíos del país y la capital, con perspectiva global, orientado a la convivencia, producción, innovación, cocreación, investigación y difusión de la cultura tecnológica.

\section{Investigación colectiva;}

El proceso de materialización basado en el Modelo para Armar se apoya en el trabajo y la investigación colectiva de los dos laboratorios que constituyen el departamento de Informática Aplicada al Diseño (Deplnfo) de la Facultad de Arquitectura, Diseño y Urbanismo (FADU) perteneciente a la Universidad de la República; por un lado el Laboratorio de Visualización Digital Avanzada (VidiaLab) con un perfil consolidado en el modelado digital y por otro lado el Laboratorio de Fabricación Digital Montevideo (LabFab_MVD) especializado en la materialización mediante tecnologías de fabricación digital. Ambos laboratorios tienen un camino recorrido en investigación conjunta y colectiva. El desafío que plantea el Modelo para Armar implica profundizar el vínculo entre ambos laboratorios dada la complejidad de la tarea encomendada en la que se produce un contínuo feedback entre digitalización/materialización; un diálogo entre software y hardware que se traduce en dimensiones y unidades de trabajo, trayectorias y velocidades de vectores y máquinas, espesores y tolerancias dimensionales de materiales y herramientas que deben ser continuamente redimensionadas en una especie de artesanía digital.-

\section{Objetivos generales y específicos}

El objetivo principal que se plantea es - a través de la integración en procesos colaborativos, de inteligencia distribuida y de investigación colectiva- la integración de tecnologías de fabricación digital en procesos de revitalización/recalificación urbana que contribuyan al desarrollo sostenible e inteligente de las ciudades.

Por otra parte, los objetivos específicos son los que se detallan a continuación;

01) integración de los dos laboratorios que conforman el Departamento de Informática Aplicada al Diseño (Deplnfo); Vidialab y fabLab en la realización de un modelo a escala urbana de la Ciudad Vieja. Cabe señalar que, si bien dicha integración ya es existente y operativa, la realización del modelo implica nuevos desafíos dada la complejidad de la maqueta a realizar proponiendo nuevas dinámicas de trabajo así como la integración de estudiantes en régimen de pasantías. Dicha complejidad está pautada, en gran medida, por el contínuo feedback entre software de modelado $3 \mathrm{~d}$ y hardware de materialización, propio de los procesos de fabricación digital. Por otra parte la curva de aprendizaje de los estudiantes colaboradores en la realización de los modelos implica un dominio gradual, a modo de expertise, en la creación de detalle -distinto caso a caso- que debe darse en dicho proceso de modelización.-

02) la materialización de la maqueta a través de las tecnologías aditiva y sustractiva, como objetivo específico en sí mismo implica la profundización del know-how existente a nivel operativo. Dadas las proporciones y cantidades de elementos a materializar, en un plazo acotado, se deben optimizar los tiempos de producción, hecho no menor 
considerando que el laboratorio de fabricación digital debe seguir realizando otras actividades de apoyo a proyectos académicos de estudiantes y talleres de Facultad de Arquitectura. Implicó la integración de software de cálculo temporal de las materializaciones para cronometrar los tiempos de ejecución.-

03) Documentación de los procesos de digitalización y materialización que posibiliten la transferencia tecnología a través de cursos de enseñanza, workshops y seminarios así como la elaboración de protocolos de uso a partir de la experiencia realizada.-

04) Creación de soporte material sobre los cuales aplicar tecnologías de visualización digital avanzada, en particular realidad aumentada y video mapping; este objetivo particular implica un upgrade con respecto al modelo original dado que se preve una instancia posterior (2.0) sujeta a un nuevo convenio con la Intendencia.-

05) Generación de un inventario digital de edificios que conforman el tejido urbano de la Ciudad Vieja. Dicho inventario, a modo de repositorio, conforma una base de datos de libre acceso, en coherencia con la política de la administración municipal.-

\section{Procedimientos}

metodológicos

Dadas las proporciones de la maqueta solicitada (2 metros * 3metros) y las consiguientes logísticas de desplazamiento y relevamiento de información, las cantidades y complejidades de elementos a materializar, los plazos acotados de ejecución, los tiempos prolongados de la administración pública en formalizar acuerdos por parte de sus departamentos jurídicos así como la la necesidad de integrar a estudiantes en régimen de pasantías en los procesos de trabajo de campo y de digitalización y materialización de datos (con los consiguientes tiempos para llamados de pasantías y procesos de transferencia tecnológica), la estrategia necesaria implica un proceso colaborativo y paramétrico, que permita anticipar, coordinar y acotar los plazos de ejecución; para ello se realiza, a través de programación gráfica, un tablero algorítmico que, a partir de la emulación de los datos cartográficos básicos de la Ciudad Vieja, permita integrar los datos relevantes para la materialización y visualización de la forma: planimetría y altimetría, edificios patrimoniales, espacios públicos, entre otros. El tablero original se disgrega operativamente en 6 sectores con un metro de ancho por un metro de largo de dimensiones, que luego se agrupan de acuerdo a una lógica $2 \times 3$, restableciendo de ese modo la dimensión original.

Si bien el proceso de materialización está pautado por una serie de acciones ordenadas las primeras etapas de obtención de información así como su procesamiento y optimización se caracteriza por un proceso de feedback ensayo-error en la que las etapas se superponen y se estabilizan en forma próxima a la finalización de todo el proceso. No obstante ello se puede identificar con claridad las siguientes etapas en la conformación del proceso de materialización:

a) relevamiento de información: obtención de datos relacionados con la altimetría y planimetría de los edificios y elementos arquitectónicos, tales como balcones, aberturas, lucernarios, claraboyas, retiros laterales, frontales 0 posteriores que componen, y enriquecen el entramado urbano; en esta instancia participan la obtención de datos mediante drones, la existencia de planos analógicos y fotos aéreas la potencialidad y versatilidad de Google street view.

b) organización de datos; previo a la digitalización de los insumos es necesario poder articular los datos obtenidos en el primer paso definiendo las escalas de intervención dadas, en gran medida, por las dimensiones del material que compone la base.

c) digitalización de modelos; la digitalización de los modelos se realiza teniendo en cuenta que serán procesados con dos tecnologías de fabricación digital; aditiva y sustractiva. Ello implica modelar, a escala 1-1 y con unidades milimétricas, las formas urbanas y arquitectónicas sin espesores. El espesor se incorpora en la etapa de optimización posterior que detallamos a continuación.

Figura 2.

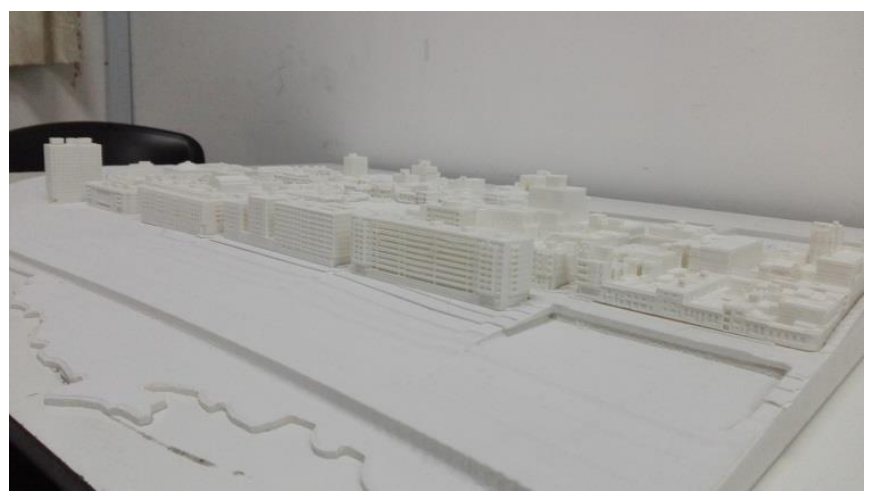

d) optimización de archivos; considerando que el proceso de fabricación digital supone la utilización de hardware con requerimientos dimensionales en cuanto a espesores, y tolerancias dimensionales, de materiales y herramientas el proceso de optimización implica la incorporación de espesores de acuerdo a la tecnología con la que será materializada; para el caso de la fabricación aditiva, si bien el espesor mínimo es de 0,1 milímetro (una micra) la correcta materialización implicó trabajar con espesores de muros de 1 milímetro (si escaláramos, mediante ingeniería inversa, esas dimensiones a los muros de un edificio genérico resultarían de 75 centímetros de espesor...). Existe por lo tanto un proceso de abstracción. En el caso de la fabricación sustractiva, la escala de trabajo está condicionada por el espesor de las 
placas en las que se "tallará" la topografía geográfica. Las "curvas" altimétricas características se procesan, en la etapa de modelización, mediante el comando $3 d$ de generación volumétrica de transición (loft) y el espesor se adapta, sucesivamente mediante operaciones booleanas de diferencia e intersección, al máximo espesor de la placa de pvc (de 20 milímetros) que será el soporte de la topografía del sitio en el que se implanta la Ciudad Vieja.

Figura 3.

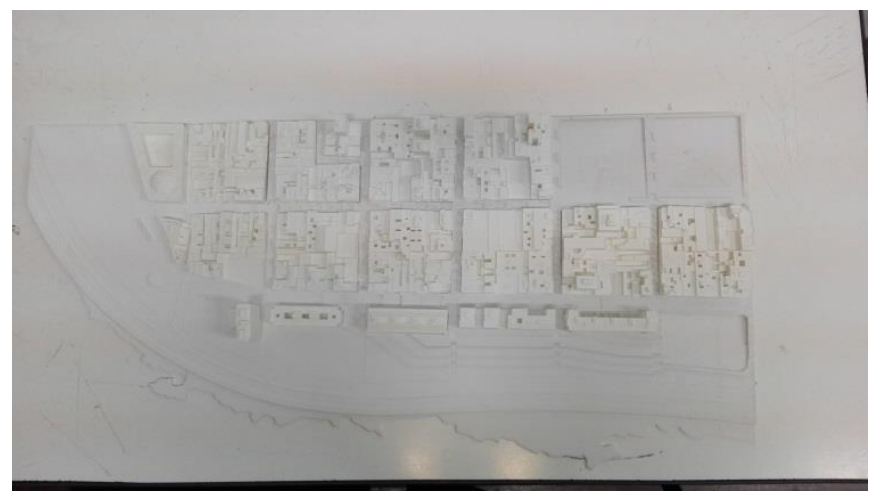

e) materialización; el proceso de fabricación digital implica la materialización del flujo de trabajo digital. Tanto para la tecnología sustractiva ( mediante router $\mathrm{cnc}$ ) como para la tecnología aditiva (impresión por deposición fundida) el insumo básico son archivos digitales basados en estereolitografía (stl.) que son "mecanizados" por medio de software del tipo slicer, el que,básicamente, se transforma las formas en información vectorial de trayectorias, velocidades, profundidades que son procesadas por máquinas cartesianas (basadas en ejes $y, x, z$ ). En el caso de la fabricación por tecnología sustractiva el proceso consiste en extraer material de la placa, asimilándose al proceso de tallado, a través de aproximaciones sucesivas; una primer instancia de desbaste general con fresas del tipo prismática y dos instancias finales de terminación a través de un proceso conocido como waterline (según ejes $\mathrm{x}$ e $\mathrm{y}$ ) realizado con fresas del tipo cabeza esférica que permite terminación superficial "lisas". Es un proceso con largos tiempos de ejecución; en total se procesan 12 placas de pvc de 1 metro cuadrado de superficie (y 20 milímetros de espesor) que implican, cada una de ellas, 36 horas discontínuas de trabajo, lo que se traduciría en $\left(12^{*} 36\right) / 8=54$ jornadas de trabajo, sin embargo la posibilidad de un trabajo contínuo de 24 horas realizado por las máquinas permite reducir considerablemente esos plazos de ejecución. En efecto, se reducen los tiempos a un tercio del total pasando a tan solo 18 días, sin embargo esa reducción conlleva riesgos dado que se pueden (y suceden)dar discontinuidades de suministro de energía eléctrica que paralizan el flujo de trabajo- Para el caso de la fabricación aditiva mediante impresión 3d el trabajo consiste en materializar las 120 manzanas que constituyen la trama urbana de la Ciudad Vieja; cada manzana, que se fabrica en forma individual, en las impresoras $3 \mathrm{~d}$ del laboratorio tiene una duración aproximada de 12 horas, lo que se traduciría en $\left(12^{*} 120\right) /\left(8^{\star} 2\right.$ impresoras)
= 90 días de trabajos. Al igual que en la planificación de flujo de trabajo de la fabricación sustractiva, se diseñan jornadas con 24 horas de duración con idénticos riesgos en cuanto suministro energético más los problemas propios de este tipo de tecnología; despegues de la mesa de impresión y atascos de la bobina de material termoplástico que resultan en el descarte total del modelo materializado.

Figura 4

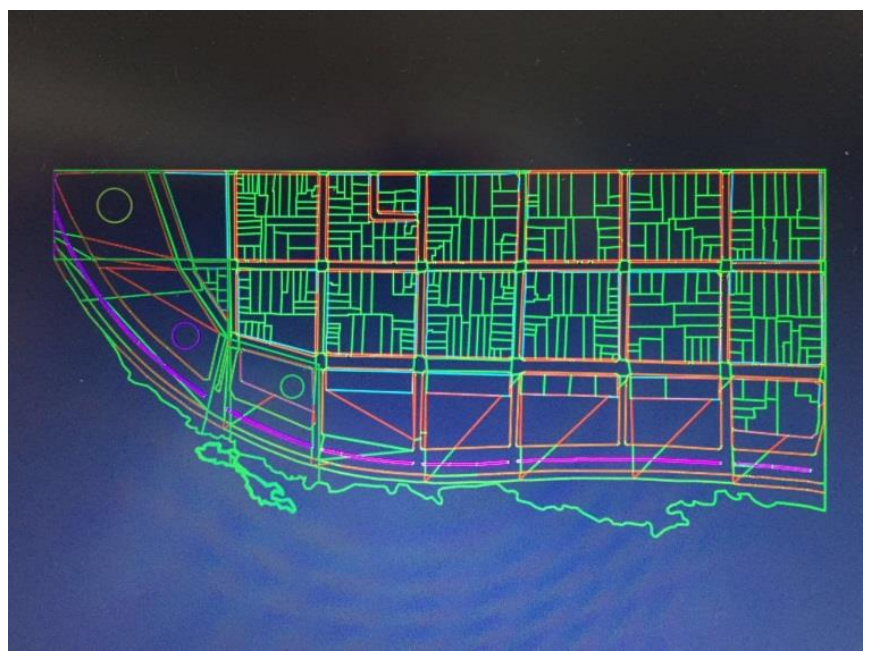

f) posicionamiento y ensamblaje; una vez realizadas las piezas mediante los procesos de fabricación descriptos anteriormente es necesario posicionar las manzanas impresas en las placas talladas que conforman la topografía (para ello se ha incorporado el perímetro de cada una de las manzanas en bajorelieve en el proceso de fabricación sustractiva). El ensamblaje trae aparejado trabajos complementarios de terminación tales como eventuales trabajos de lijado y /o limado de superficies lo que se realizan, por razones locativas, en las instalaciones del Laboratorio de Visualización Digital.

g) traslado definitivo; dado que el modelo para armar será exhibido en un lugar diferente al que fue armado y ensamblado se debieron tener en cuenta detalles tales como anchos de aberturas que posibilitaran su traslado así como también el peso parcial de cada estructura dado que no se iban a disponer de elementos mecánicos auxiliares de movimiento vertical, como ascensores o montacargas, para su traslado hacia su destino final: las instalaciones del Laboratorio de Innovación Social sito en el Cabildo de Montevideo.-

\section{Resultados}

Si bien el resultado esperado es la materialización de una maqueta de escala urbana mediante tecnologías de modelización y fabricación digital, dado el pedido realizado por 
parte del organismo gubernamental, se buscará la obtención de resultados relacionados:

a) profundización de conocimientos de Tecnologías de Informática Aplicada; cuando hablamos de las tecnologías de fabricación digital estamos en presencia de dos grandes grupos o "modus operandi" de materialización; la tecnología "sustractiva" que consiste en retirar parte de la materia ( por medios térmicos o mecánicos) y la tecnología "aditiva" que consiste en adicionar (por medios puntuales, lineales 0 superficiales) materia.- En este grupo de conocimientos, adquiridos y profundizados, debemos destacar los avances logrados en las instancias de mecanizado de ambas prácticas ya sea en la optimización de los modelos así como de los tiempos de ejecución, que redunda en mejor utilización de los recursos humanos e insumos materiales aplicados en la consecución de objetivos; cuando hablamos de las tecnologías de visualización digital avanzadas estamos en presencia de un conjunto de tecnologías que implican el adecuado manejo de drones así como la obtención de modelos digitales por técnicas de ingeniería inversa que incluyen, entre otras; la rotoscopía, la hibridación de medios analógicos y digitales (conjugando fotos aéreas y frontales con planimetrías a través de homotecias de igualación).-

b) integración de las funciones universitarias de enseñanza, investigación y extensión en proyectos de emponderamiento ciudadano y responsabilidad social que contribuyan al desarrollo sostenible e inteligente de las ciudades apoyando iniciativas ad hoc. Este punto, que será tratado con mayor profundidad en la discusión de aporte de la investigación, trae aparejado como resultado relacionado la conformación de una Laboratorio de Innovación Ciudadana que se servirá de la maqueta, como modelo para armar, en el mapeo de iniciativas de innovación ciudadana y de las tecnologías de Informática Aplicada en el prototipado de dichas iniciativas.

c) conformación de un inventario urbano-arquitectónico que, a modo de repositorio digital, permitan generar una base de datos de libre acceso, en coherencia con la política de la administración municipal. Dicho repositorio, gestionado como un "work in progress", es posibilitado por la concepción paramétrica del modelado digital desarrollado en la conformación de los padrones y manzanas que integran el tejido urbano de la intervención. En dicha concepción cada modelo es una "instantánea" de un proceso en continuo desarrollo. El inventario urbano-arquitectónico no solo incorpora información catastral típica como ancho y largo de los terrenos, factor de ocupación del suelo, retiros y las alturas de edificación sino también las características volumétricas, la relación lleno-vacío, la presencia de balcones y otras singularidades arquitectónicas que, perteneciendo a la tipología arquitectónica caracterizan y enriquecen al tejido urbano.

\section{Discusión}

La importancia de la presente investigación radica en la integración de las potencialidades de la Informática Aplicada (tecnologías de fabricación digital y de visualización digital avanzadas) no tanto como poderosos recursos instrumentales (que sin lugar a duda lo son) sino como apropiación y participación de nuevas estrategias colaborativas en proyectos de mayor responsabilidad social que contribuyan al desarrollo sostenible e inteligente de las ciudades.

En tal sentido, la integración de la maqueta como objeto de investigación, de materialización y de visualización en un Laboratorio de Innovación Ciudadana constituye un primer hito hacia la integración y empoderamiento de la ciudadanía mediante procesos de Inteligencia Distribuida, intrínsecos a este tipo de laboratorios. Como lo define el documento colaborativo Lab ciudadanos, elaborado por la SEGIB (Secretaría General Iberoamericana); "Los laboratorios ciudadanos son espacios en los que las personas con distintos conocimientos, habilidades y diferentes grados de especialización académica y/o práctica se reúnen para desarrollar proyectos en común. Espacios que exploran las formas de experimentación y aprendizaje colaborativo que han surgido de las redes sociales para impulsar procesos de innovación ciudadana. Desde la perspectiva del proceso de la Innovación Ciudadana, estos proyectos trabajados y generados en laboratorios ciudadanos tienen la característica de buscar una transformación social, que contribuya al desarrollo cultural, social y económico de nuestros países".

Por otra parte y dado el hecho de que el Laboratorio de Innovación Ciudadana se va a localizar en las instalaciones del Cabildo de Montevideo, ubicado en la Ciudad Vieja, hay un interesante proceso de resemantización de dicha institución que podríamos caracterizar como Ciudadanía 2.0, en plena sintonía con los preceptos que establece la SEGIB. En efecto el edificio del Cabildo y Reales Cárceles constituye uno de los monumentos más representativos del Montevideo colonial. Declarado Monumento Histórico, su construcción data de 1804 según proyecto original de Tomás Toribio $\left(1^{\mathrm{er}}\right.$ arquitecto español que ejerció la disciplina en Uruguay). El Cabildo, que albergó en un principio funciones capitulares y dependencias carcelarias, como lo establecía la Legislación de las Indias, se constituyó en un emblema de Montevideo y de Uruguay. Por casi cien años fue el reducto del primer gobierno comunal de la ciudad y en sus salas se sancionó y juró un 18 de Julio de 1830 la primer Constitución de la República, funcionando posteriormente el Poder Legislativo y el Ministerio de Relaciones Exteriores, entre otras instituciones .El 21 de setiembre de 1958, se inauguró en este edificio el Museo y Archivo Histórico Municipal. La actual intervención, al igual que la maqueta, nuestro modelo para armar, conjuga pasado, presente y futuro en la resemantización del edificio mediante el empoderamiento de la ciudadanía a través de innovaciones que permitan un desarrollo sostenible y basado en la inclusión social. La maqueta formará parte del equipamiento del Laboratorio de 
Innovación Ciudadana permitiendo la visualización de las intervenciones pasadas, presentes y futuras así como de las nuevas posibilidades materiales de las tecnologías de fabricación digital y de observación de las tecnologías de visualización digital ya que será soporte (en etapas posteriores) de técnicas de realidad aumentada y video mapping.

Otro aspecto importante de señalar es el relacionado al papel que ocupa la Universidad en este proceso colaborativo, de inteligencia distribuida e investigación colectiva dado que las funciones universitarias de investigación, enseñanza y extensión, consagradas en la Carta Orgánica, no son fines en sí mismos sino instrumentos para lograr que Universidad de la República pueda contribuir al desarrollo nacional a través de un diálogo permanente con la sociedad. En tal sentido la presente investigación, en pleno proceso de desarrollo, permite la consecución de tales fines al integrar como instrumentos la investigación, la enseñanza y la extensión universitaria.-

\section{Agradecimientos}

Quiero agradecer el apoyo y colaboración de todos los integrantes del VidialLab y del LabFab, laboratorios integrantes del Departamento de Informática Aplicada y en especial a su director, arquitecto Marcelo Payssé.-

\section{Referencias}

Beorkrem, C. (2012). Material Strategies in Digital Fabrication, NY: Replika Press.

Hauschild, M. (2012). Digital Processes, Munich: Birkhäuser Press.

Rodríguez Barros, Diana (2004) Hipermedios y modelos virtuales de fragmentos urbanos.

Lima, F; Ripper Kós,J; Pensamento algorítmico, parametrizacao e urbanismo sustentavel; uma avaliacao de parámetros para estrategias de projeto urbano inteligente. Proceedings of the 18th Congress of the Iberoamerican Society of Digital Graphics.

$<$ Kolarevic, Branko. 2003. Architecture in the digital age; design and manufacturing. New York, Spon Press.

Gutiérrez, M.; Pérez de Lama et al, FabWorks (2011). Diseño y fabricación digital para la arquitectura, Escuela Técnica Superior de Arquitectura Universidad de Sevilla

Iwamotto, Lisa; (2009) Digital Fabrications. Architectural and Material Techniques, Princeton Architectural Press, Nueva York;

Segib; Secretaría General Iberoamericana (2013) Laboratorios Ciudadanos; espacios para la innovación ciudadana, http://www.ciudadania20.org 